\title{
Lifelong Voluntary Exercise Modulates Age-Related Changes in Oxidative Stress
}

\author{
Authors \\ Mohamed Amine Bouzid ${ }^{1}$, Edith Filaire ${ }^{2}$, Régis Matran ${ }^{3}$, Sophie Robin ${ }^{3}$, Claudine Fabre ${ }^{4}$
}

\section{Affiliations}

1 Unité de Recherche Education, Motricité, Sport et Santé UR15JS01, Institut Supérieur du Sport et de l'Education Physique de Sfax, Université de Sfax, Tunisie

2 Laboratoire CIAMS, EA4532, Université Paris-Sud, Université Orléans, Orléans, France

3 Service EFR, CHRU de Lille, Boulevard du Pr Leclercq 59037 Lille, France.

4 Université de Lille, URePSSS, EA 7369 " Activité Physique Muscle Santé ", Faculté des Sciences du Sport et de l'Education Physique, 9 rue de l'Université, 59790 Ronchin, France

\section{Key words}

aging, oxidative stress, exercise, physical activity

accepted 05.09.2017

\section{Bibliography}

DOI https://doi.org/10.1055/s-0043-119882

Published online: 23.11 .2017

Int J Sports Med 2018; 39: 21-28

(c) Georg Thieme Verlag KG Stuttgart · New York

ISSN 0172-4622

\section{Correspondence}

Mr. Mohamed Amine Bouzid

Universite de Sfax, Unité de Recherche Education

Motricité, Sport et Santé UR15JS01

Institut Supérieur du Sport et de l'Education Physique de Sfax 3029, Sfax

Tunisia

Tel.: + 216/22/06/22/75, Fax: + 33/3/20887363

bouzid.mohamed-amine@hotmail.fr

\section{ABSTRACT}

The hypothesis that aging and regular physical activity could influence oxidative stress has been studied by comparing antioxidant activities (superoxide dismutase (SOD), glutathione peroxidase (GPX), glutathione reductase (GR), ascorbic acid and $\alpha$-Tocopherol) and malondialdehyde level (MDA) in four groups: young sedentary ( $n=15$; age: $20.3 \pm 2.8$ years; $Y S)$, young active ( $n=16$; age: $21.4 \pm 1.9$ years; $Y A)$, old sedentary $(n=15$; age: $65.1 \pm 3.5$ years; OS) and old active ( $n=17$; age: $67.2 \pm 4.8$ years; $\mathrm{OA})$. Antioxidant activities and MDA level were assessed at rest and after an incremental exercise. There was no difference in resting antioxidant activities and lipid peroxidation between YS and OS. However, resting SOD and GR activities were higher in YA compared to $O A(p<0.01$ and $p<0.05$, respectively) and resting MDA level was higher in OA compared to $Y A(p<0.01)$. After exercise, a significant increase in SOD and $G P X$ activities was observed in YS, YA and OA $(p<0.01)$. Likewise, after exercise a significant increase of MDA level in YA, OS and $O A(p<0.01)$ was observed. In addition, the comparison of $Y A$ to $O A$ and $Y S$ to $O A$ revealed similar antioxidant activities and lipid peroxidation between $Y S$ and $O A$, whereas antioxidant activities were higher in YA compared to OA. These data suggest that beneficial effects of regular physical activity in antioxidant defense and lipid peroxidation damage could be impaired by the aging process and that regular physical activity in older adults could maintain age-related decreases in antioxidant defense.

\section{Introduction}

Aging is a multifactorial and complex process characterized by a decline in the ability to respond to stress and to maintain cellular and systemic homeostasis. The free-radical theory of aging has been the basis of the major anti-aging strategies, although this theory was not yet been definitely confirmed [31]. In fact, among the various theories that attempt to explain the aging process, disruption of the whole signaling network involving reactive oxygen species (ROS) has received increasing recognition over the past two decades [47].
These species play a very important role in the changes induced by aging in various tissues [14]. ROS accumulation, including superoxide anion, hydrogen peroxide, and the subsequent oxidative damage to cells can be neutralized to nontoxic forms via elevated antioxidant defenses, which include superoxide dismutase (SOD), catalase (CAT), glutathione peroxidase (GPX), and glutathione reductase (GR) $[26,49]$. Non-enzymatic antioxidants include a variety of free radical quenchers, such as ascorbic acid, alpha-tocopherol, carotenoids, flavonoids, thiols (which include GSH), ubiquinone Q10, uric 
acid, bilirubin, ferritin, albumin, transferrin, lactoferrin and micronutrients, which act as enzymatic cofactors. The aging process causes an imbalance between ROS production and antioxidant capacity of the tissues, leading to cell damage [7]. Therefore, further studies should investigate the activation of the host defense mechanisms in response to oxidative stress in older adults.

Apparently, one of the mechanisms for activating such responses is stress itself [20]. In this context, several studies showed that physical exercise could induce positive stress [17, 36, 37]. In fact, it has been reported that a single bout of exercise induces oxidative stress while increasing antioxidant enzyme activities in both young and elderly subjects [39]. Likewise, animal studies have reported that gene expression and protein content can be upregulated by a single bout of exercise $[21,32]$. These responses to the stimulus of exercise can be attributed to redox signaling leading to activation of pathways involved in transcription of antioxidant gene enzymes and increasing resistance to cellular stress [8, 24, 43]. The length of time that these effects last from a single bout of exercise is not well characterized, but it is likely limited unless the stimulus is applied repeatedly as would occur with chronic exercise. In this sense, recent findings reported a significant improvement in oxidant/antioxidant balance in response to chronic physical activity through enhancing the endogenous antioxidant defense system in young and older people [2, 30]. In fact, ROS produced during exercise could be harmful to unprepared tissues but may also activate adaptive responses to oxidative stress, inducing antioxidant defense systems by upregulation of responsible gene expression [16]. However, it is still unknown whether these adaptive responses differ in elderly and young subjects. It could be hypothesized that the endogenous antioxidant system in exercising elderly subjects is not relevant for the same adaptive responses as compared to exercising young subjects.

On the other hand, significant changes in antioxidant capacity due to regular physical activity might be more easily detected by examining the effect of exercise on response to an acute oxidative challenge, which provides a more robust test of an individual's antioxidant capacity [46].Previous studies that have measured oxidative stress responses under challenge conditions have generally used acute exercise as a physiological challenge [12, 30, 41]. In response to acute physical exercise, several studies reported better antioxidant defenses and a lower oxidative stress damage level in the young compared to the elderly. However, less is known about the effects of regular physical activity on exercise-induced oxidative stress in elderly and young subjects. In others words, to the best of our knowledge, data on oxidative stress and acute exercise have mainly focused on parameters measured immediately before and after an exercise bout. However, it is not well known whether these responses to chronic exercise provide sustained protection against subsequent oxidative stressors, especially in older subjects.

Therefore, the aim of this study was to investigate both aging and regular physical activity effects on blood antioxidant activities and lipid peroxidation levels, as markers of oxidative stress, in healthy young and older subjects at rest and in response to acute exercise. We put forward the hypothesis that regular physical activity (i) could maintain an aging-associated decrease in antioxidant defenses and an increase in lipid peroxidation damage, and (ii) that beneficial effects on oxidative stress could be impaired by the aging process.

\section{Methods}

\section{Participants}

A total of 78 men and women were pre-screened. Twelve were excluded from the study due to the exclusion criteria [i. e., active coronary artery disease $(n=3)$, hypertension $(n=3)$, hormonal replacement therapy $(n=2)$, and diabetes $(n=4)$ ]. Exercise testing was completed on 66 participants and 3 of them were excluded because of concerns with blood draws. Thus, 63 healthy participants were included to the data analysis. Characteristics of the subjects are presented in $>$ Table $\mathbf{1}$.

All participants were non-smokers, free of musculoskeletal problems and orthopedic/neuromuscular limitations, had a resting blood pressure below $140 / 85 \mathrm{~mm} \mathrm{Hg}$, had no sign of cardiovascular/respiratory complications at rest or during the progressive diagnostic graded exercise test and did not use hormonal replace-

- Table 1 Descriptive data of the participants (mean \pm SD).

\begin{tabular}{|c|c|c|c|c|}
\hline & $\begin{array}{l}\text { Young sedentary } \\
\text { group } N=15\end{array}$ & $\begin{array}{l}\text { Young active group } \\
\qquad \mathrm{N}=16\end{array}$ & $\begin{array}{l}\text { Old sedentary group } \\
\qquad \mathrm{N}=15\end{array}$ & $\begin{array}{l}\text { Old active group } \\
\qquad \mathrm{N}=17\end{array}$ \\
\hline Age (years) & $20.3 \pm 2.8$ & $21.4 \pm 1.9$ & $65.1 \pm 3.5^{* * \# \#}$ & $67.2 \pm 4.8^{* * \# \#}$ \\
\hline Weight (Kg) & $66.1 \pm 11.7$ & $68.9 \pm 4.1$ & $71.8 \pm 7.6$ & $72.2 \pm 8.5$ \\
\hline Height (m) & $1.7 \pm 0.2$ & $1.7 \pm 0.3$ & $1.6 \pm 0.1$ & $1.7 \pm 0.1$ \\
\hline Physical activity score & $3.8 \pm 1.1^{* *}$ & $16.9 \pm 2.2$ & $4.1 \pm 2.3 \pi$ & $19.4 \pm 2.4$ \\
\hline $\mathrm{VO}_{2 \max }(\mathrm{ml} / \mathrm{min} / \mathrm{kg})$ & $41.2 \pm 3.2^{* *}$ & $58.2 \pm 8.1$ & $23.2 \pm 4.4^{* *}$ "†\#\# & $40.6 \pm 3.9^{* *}$ \\
\hline MAP (Watts) & $220.7 \pm 36.4^{* *}$ & $296 \pm 27.9$ & $94.1 \pm 32.6^{* *}$ *\#\# & $195.9 \pm 27.2^{* *}$ \\
\hline $\mathrm{HR}_{\text {max }}$ (bpm) & $185.2 \pm 9.1$ & $188.3 \pm 5.9$ & $148 \pm 19.3^{*} \#$ & $150.4 \pm 16.7^{*} \#$ \\
\hline $\begin{array}{l}\text { Time duration of incremental } \\
\text { exercise (min) }\end{array}$ & $19.2 \pm 3.6$ & $17.4 \pm 2.8$ & $16.8 \pm 5.4$ & $16.4 \pm 2.8$ \\
\hline \multicolumn{5}{|c|}{${ }^{*}$ : Significantly different from YA $(\mathrm{p}<0.05),{ }^{*}{ }^{*}$ : Significantly different from YA $(\mathrm{p}<0.01)$} \\
\hline \multicolumn{5}{|c|}{ \#: Significantly different from YS ( $p<0.05), \#$ : Significantly different from YS $(p<0.01)$} \\
\hline \multicolumn{5}{|c|}{ †: Significantly different from OA $(p<0.05)$, $\dagger$ : Significantly different from $O A(p<0.01)$} \\
\hline \multicolumn{5}{|c|}{$\mathrm{VO}_{2 \max }$ : maximal oxygen uptake, MAP: maximal aerobic power, $\mathrm{HR}_{\max }$ : maximal heart rate } \\
\hline
\end{tabular}


ment therapy or supplementation with antioxidants within the 3 months preceding the study.

All the participants were informed about the purpose, nature, and potential risks related to the study and gave their written informed consent before participation. The study protocol was approved by the Ethics Committee of CHRU of Lille ( ${ }^{\circ}$ 07/42) and met the ethical standards of the International journal of Sports Medicine [18].

Participants were assigned to one of four groups according to their age and physical activity level:

a) young sedentary (YS, 20.3 $\pm 2.8 \mathrm{yrs}, \mathrm{N}=15)$,

b) young physically active (YA, $21.4 \pm 1.9 \mathrm{yrs}, \mathrm{N}=16$ ),

c) old sedentary (OS, 65.1 $\pm 3.5 \mathrm{yrs}, \mathrm{N}=15)$, and

d) old physically active $(\mathrm{OA}, 67.2 \pm 4.8 \mathrm{yrs}, \mathrm{N}=17)$.

To be included in the study, the age range for the young participants was $18-30$ years and at least 60 years for elderly participants.

Participants were assigned to the active or sedentary groups based on the following criteria: (i) Maximal oxygen uptake $\left(\mathrm{VO}_{2 \max }\right)$ had to be below $25 \mathrm{ml} / \mathrm{kg} / \mathrm{min}$ and $45 \mathrm{ml} / \mathrm{kg} / \mathrm{min}$ for OS and $\mathrm{YS}$ subjects, respectively. For OA and YA participants, and according to the American College of Sports Medicine [1], $\mathrm{VO}_{2 \max }$ values had to be over $35 \mathrm{ml} / \mathrm{kg} / \mathrm{min}$ and over $45 \mathrm{ml} / \mathrm{kg} / \mathrm{min}$, respectively.

(ii) Lifetime physical activity history was assessed using the physical activity questionnaire of Baecke et al. [4] for young subjects and the physical activity questionnaire of Voorrips et al. [48] for elderly participants, both of which were adapted to each age category. Participants were interviewed about the frequency of the physical activities they performed during the last 12 months. To be included in the present study, physical activity scores in the Baecke et al. questionnaire [4] had to be below 6.0 for the YS and over 9.0 for YA. For OS and OA, scores in the Voorrips et al. physical activity questionnaire [48] had to be below 9.0 and over 16.0, respectively.

YS and OS were recruited from social or cultural clubs in and around the city of Lille, France. However, YA and OA were recruited from the French Federation of "Cyclotourisme." They must have had more than two consecutive years of cycling exercise for at least 10 months per year and no less than $10 \mathrm{~h} /$ week. They performed between 60 and $80 \mathrm{~km}$ of cycling per session and three times per week.

\section{Procedure}

On arrival of each participant at the hospital (CHRU of Lille, France), a clinical interview, a resting electrocardiogram (ECG), and a record of blood pressure were carried out to verify the exclusion criteria of the protocol. Subsequently, all participants filled out a questionnaire about their physical activity practices and their daily food intake. Then, body weight and height were measured. Body mass was recorded to the nearest $0.1 \mathrm{~kg}$ using a portable digital scale (PreciaMolen, I 200B, Precia SA, Privas, France). Standing height was measured to the nearest $0.1 \mathrm{~cm}$ with an anthropometric plane. After anthropometric measurements, a nurse inserted a venous catheter in the forearm of the participant to take a resting blood sample. Then, each participant performed an incremental exercise test until exhaustion, followed by a period of recovery for $10 \mathrm{~min}$, when post-exercise blood samples were collected again.

\section{Exercise protocol}

Participants were asked to avoid strenuous physical activity during $48 \mathrm{~h}$ before the exercise testing session. Each subject performed an incremental exercise test until exhaustion on an electricallybraked cycle ergometer (Excalibur Sport, Lode B.V, Medical Technology, Groningen, Netherlands) connected to a computer with diagnostic software (Ergocard $\AA$, Medisoft, Dinant, Belgium). After resting for 3 min on the cycle ergometer, the participant started exercise at 30 watts $(\mathrm{W})$ and 60-70 revolutions per minute of pedaling for $3 \mathrm{~min}$ as a warm-up period. Then, the exercise was performed with an increased workload of $20 \mathrm{~W}$ for men and $10 \mathrm{~W}$ for women every 3 min for the OS group. For YS and OA subjects, the workload increased by $30 \mathrm{~W}$ every 3 min until the end of the exercise. However, for the YA group, the workload increased by $30 \mathrm{~W}$ every 2 min. For all subjects, the last completed stage was followed by $2 \mathrm{~min}$ of active recovery at $25 \mathrm{~W}$ and $3 \mathrm{~min}$ of passive recovery. The workload increases were chosen in order to ensure equality in exercise testing duration between the groups. Gas exchanges (oxygen consumption: $\mathrm{VO}_{2}$, carbon dioxide production: $\mathrm{VCO}_{2}$, and ventilation: $\mathrm{VE}$ ) were measured continuously throughout the test and for 5 min during the post-exercise period using a gas-exchange system analyzer (Medisoft, Belgium). Blood pressure was measured at rest and every 3 min during and after the exercise period.

The exercise testing was finished when: (i) exhaustion of the participant or inability to maintain the required pedaling speed was observed, (ii) the $\mathrm{VO}_{2}$ plateau was reached, and (iii) predicted maximum heart rate (HR max) was reached (208-0.7 × age $\pm 10 \%$ ) [44].

\section{Dietary record}

All participants received a detailed verbal explanation and written instructions. Food intake was recorded one week before the beginning of the study and over 4 days including one weekend day to account for variations that might occur between weekdays and weekends [22]. Participants were asked to maintain their usual dietary habits during the recording period and to be as accurate as possible in registering the amount and type of food and fluid consumed. They were asked to record brand names of all commercial and ready-to-eat foods and the methods of preparation. A list of common household measures, such as cups and tablespoons, and specific information on the quantity in each measurement (grams, etc.) was given to each participant. Any questions, ambiguities, or omissions regarding the type and amount of food and beverages consumed were resolved during individual interviews. Each individual's diet was assessed using the Nutrilog software package (2.31), a computerized database (Proform) that calculates food composition from the French standard reference in relation to age [11].

\section{Blood sampling}

A blood sample was drawn at rest and 5 min following the exercise to measure MDA level and 20 min after the exercise to measure SOD, GPX, GR, ascorbic acid, and $\alpha$-Tocopherol levels. We chose to take blood samples at different time points because it has been reported that post-exercise lipid peroxidation peak is generally obtained before higher antioxidant enzyme values [29].

In our study, blood was drawn from the antecubital vein into a dry tube for the measurement of $\alpha$-Tocopherol, an EDTA tube for the measurement of ascorbic acid and MDA, and a heparinized tube 
for the measurement of SOD, GPX, and GR. Whole blood was centrifuged at $3000 \mathrm{rpm}$ for $10 \mathrm{~min}$ at $4^{\circ} \mathrm{C}$ and plasma was removed. Erythrocytes were washed three times with $0.9 \% \mathrm{NaCl}$ solution (normal saline) at $4{ }^{\circ} \mathrm{C}$. Thereafter, $2 \mathrm{ml}$ of cold distilled water was added to the erythrocytes. Then, to achieve complete lysis of erythrocytes, samples were stored at room temperature for 5-10 min. Finally, the mixture was centrifuged for $10 \mathrm{~min}$ at $4^{\circ} \mathrm{C}$. The clear supernatant solution was then divided into equal volumes of $0.5 \mathrm{ml}$. Aliquots of the resulting plasma and blood erythrocytes were stored at $-80^{\circ} \mathrm{C}$ until analyzed. $\alpha$-Tocopherol, ascorbic acid, and MDA levels were evaluated in plasma and SOD, GPX, and GR activities were evaluated in blood erythrocytes.

\section{Antioxidant enzyme activity assay}

SOD activity was analyzed using a clinical chemical analyzer (Konelab 60, Thermo Fisher Scientific Society). This method uses xanthine and xanthine oxidase to generate superoxide radicals that react with 2-(4-iodophenyl-)-3-(4-nitrophenyl)-5-phenyltetrazoluim chloride to form a red formazan dye. The SOD activity is then quantified by measuring the degree of inhibition of this reaction.

GPX activity was measured using a reagent set (Ransel, Randox Laboratories) and indirectly by a coupled reaction with GR. Oxidized glutathione (GSSG), produced upon reduction of an organic hydroperoxide by GPX, is recycled to its reduced state by GR and nicotinamide adenine dinucleotide phosphate (NADPH). The oxidation of NADPH to $\mathrm{NADP}^{+}$is accompanied by a decrease in absorbance at $340 \mathrm{~nm}$. The rate of decrease is directly proportional to the GPX activity in the sample.

GR activity was measured using a clinical chemical analyzer (Konelab 60). GR is a flavoprotein that catalyses the reaction, whereby reduced NADPH converts GSSG to reduced glutathione (GSH). We evaluated this enzymatic activity with a GR, determining the rate of NADPH oxidation. The oxidation of NADPH to NADP+ results in a decreased absorbance at $340 \mathrm{~nm}$ that is directly proportional to the GR activity.

\section{$\alpha$-Tocopherol}

Plasma $\alpha$-Tocopherol was measured using the high-performance liquid chromatography technique with spectrophotometric detection. In this technique, $\alpha$-Tocopherol is extracted with heptanes after protein precipitation by ethanol and then injected into the HPLC system. The selected wavelength was $292 \mathrm{~nm}$.

\section{Ascorbic acid}

After deproteinization of plasma with metaphosphoric acid, ascorbic acid was oxidized to dehydroascorbic acid by ascorbate oxidase (DHAA). The DHAA bound to the o-phenylenediamine (OPDA), which produced a chromophore whose absorbance was read at $340 \mathrm{~nm}$ on a clinical chemical analyzer (Konelab 60). Thus, the rate of ascorbic acid in plasma was deduced from the amount of chromophore formed.

\section{Lipid peroxidation}

The MDA was measured in plasma by the HPLC technique with fluorimetric detection. It was determined by modified thiobarbituric acid (TBARS). $100 \mu$ l of plasma was precipitated with trichloroacetic acid $(200 \mu \mathrm{l})$ and mixed with $450 \mu \mathrm{l}$ of normal saline solution
$(0.9 \%)$. The whole mixture was incubated in a $90^{\circ} \mathrm{C}$ water bath for $30 \mathrm{~min}$ then cooled in water. After centrifugation at $3000 \mathrm{rpm}$ for $5 \mathrm{~min}$ at $4^{\circ} \mathrm{C}$, the absorbance was read at $532 \mathrm{~nm}$.

For MDA, ascorbic acid and $\alpha$-Tocopherol assays, plasma volume was corrected using the guidelines provided by Dill et al. [10].

\section{Statistical analyses}

The statistical analysis was performed using Sigma STAT software. Normal Gaussian distribution of the data was verified by the Shapiro-Wilk test. Results were expressed as means \pm SD and $p<0.05$ was considered statistically significant. Experimental analysis for oxidative stress markers was performed with three-way ANOVA (age $\times$ physical activity status $\times$ time) to determine differences within and between groups over time. If a significant interaction was found, a Bonferroni post hoc test was used to determine if and where there was a difference between groups. To compare cardiorespiratory adaptations to the exercise, anthropometric values, and dietary intake data between groups, a one-way ANOVA test was performed.

\section{Results}

\section{Physiological exercise data and dietary intake}

The physical activity score showed a significant difference between active and sedentary subjects $(p<0.01)(\triangleright$ Table 1$)$. The assessment of $\mathrm{VO}_{2 \max }$ and maximal aerobic power (MAP) values showed that regular physical activity increased $\mathrm{VO}_{2 \max }$ and MAP in both groups (young and older) $(p<0.01)$. It should be noted that no difference in aerobic fitness capacity $\left(\mathrm{VO}_{2 \max }\right.$ and MAP) were noted between YS and OA groups ( $\triangleright$ Table 1).

Concerning the dietary analysis, and in comparison with sedentary subjects, whatever their age, exercising participants had higher daily total energy and copper intake $(p<0.05)$. YA subjects had significantly higher protein and vitamin $\mathrm{E}$ intake in comparison with the other groups $(p<0.05)$. The daily energy intake derived from carbohydrates was higher in OA compared to the other groups $(p<0.05)$. No significant difference in vitamin $C$, zinc $(Z n)$, selenium (Se) and lipid intake was shown between the studied groups ( Table 2).

\section{Oxidative stress markers}

Effects of the aging process

Resting SOD, GR, GPX, ascorbic acid, $\alpha$-Tocopherol, and MDA levels were not different between sedentary groups ( $>$ Table 3 ). Regarding active groups, resting SOD and GR activities were higher in YA compared to OA ( $p<0.01$ and $p<0.05$, respectively). However, resting MDA level was higher in OA compared to YA $(p<0.01)$ ( $\triangleright$ Table 3).

\section{Effects of regular physical activity}

The comparisons of YS to YA and OS to OA revealed that active individuals in both age groups have higher enzymatic and non-enzymatic antioxidant levels ( $\triangleright$ Table 3). Thus, YA and OA have higher antioxidant activities (SOD and GPX) and $\alpha$-Tocopherol levels in comparison with YS and OS, respectively $(p<0.01)$. 
- Table 2 Total daily energy intakes, macronutrient, and antioxidant micronutrient consumption in all groups (mean \pm SD).

\begin{tabular}{|c|c|c|c|c|}
\hline & Young sedentary group & Young active group & Old sedentary group & Old active group \\
\hline & $N=15$ & $N=16$ & $N=15$ & $N=17$ \\
\hline Energy intake (Mj/day) & $9.7 \pm 2.3^{* \dagger}$ & $13.2 \pm 2.1$ & $8.9 \pm 2.3^{* \dagger}$ & $12.4 \pm 1.7$ \\
\hline Carbohydrates (g/day) & $253.2 \pm 66^{\dagger}$ & $280 \pm 32.1^{\dagger}$ & $264.7 \pm 49.3^{\dagger}$ & $348.4 \pm 77$ \\
\hline Proteins (g/kg(BW)/j) & $1.4 \pm 0.4^{*}$ & $2.7 \pm 0.3$ & $1.6 \pm 0.9^{*}$ & $1.46 \pm 0.3^{*}$ \\
\hline Lipids (g/day) & $96.1 \pm 33.1$ & $109 \pm 33.1$ & $111.2 \pm 24.3$ & $108.5 \pm 37.5$ \\
\hline Vitamin C (mg/day) & $90.1 \pm 34.7$ & $109 \pm 20.1$ & $101.4 \pm 27.1$ & $103.2 \pm 17.8$ \\
\hline Vitamin E (mg/day) & $7.5 \pm 2.2^{*}$ & $12.9 \pm 2.1$ & $8.5 \pm 3.6^{*}$ & $9.8 \pm 2.1^{*}$ \\
\hline Selenium ( $\mu \mathrm{g} /$ day) & $65.3 \pm 12.1$ & $75.1 \pm 6.6$ & $74.1 \pm 19.2$ & $69.1 \pm 19.5$ \\
\hline Zinc (mg/day) & $8.9 \pm 1.8$ & $13.4 \pm 3.8$ & $11.2 \pm 4.3$ & $13.5 \pm 4.7$ \\
\hline Copper (mg/day) & $1.1 \pm 0.3^{* \dagger}$ & $1.8 \pm 0.7$ & $1.2 \pm 0.5^{* \dagger}$ & $1.7 \pm 0.5$ \\
\hline \multicolumn{5}{|c|}{${ }^{*}$ : Significantly different from YA $(p<0.05),{ }^{*} *$ : Significantly different from $Y A(p<0.01)$} \\
\hline \multicolumn{5}{|c|}{ \# : Significantly different from YS $(p<0.05)$, \#\# : Significantly different from YS $(p<0.01)$} \\
\hline \multicolumn{5}{|c|}{ †: Significantly different from OA ( $p<0.05)$, } \\
\hline \multicolumn{5}{|l|}{ Mj/day: megajoule/day } \\
\hline BW: body weight & & & & \\
\hline
\end{tabular}

- Table 3 Blood antioxidant status and malondialdehyde level at rest and during the post-exercise period in the 4 groups.

\begin{tabular}{|c|c|c|c|c|c|}
\hline & & $\begin{array}{c}\text { Young sedentary } \\
\text { group }\end{array}$ & $\begin{array}{l}\text { Young active } \\
\text { group }\end{array}$ & $\begin{array}{l}\text { Old sedentary } \\
\text { group }\end{array}$ & Old active group \\
\hline & & $N=15$ & $N=16$ & $N=15$ & $\mathrm{~N}=17$ \\
\hline \multirow{2}{*}{ Ascorbic acid ( $\mu \mathrm{mol} / \mathrm{l})$} & Rest & $50.7 \pm 19.1$ & $47.2 \pm 12.3$ & $43.3 \pm 20.9$ & $52.2 \pm 14.4$ \\
\hline & Post-exercise & $53.7 \pm 18.7$ & $52.9 \pm 8.5$ & $48.6 \pm 24.2$ & $54.7 \pm 16.0$ \\
\hline \multirow{2}{*}{$\alpha$-Tocopherol $(\mu \mathrm{mol} / \mathrm{l})$} & Rest & $38.7 \pm 6.1^{* *}$ & $44.4 \pm 7.8$ & $34.4 \pm 7.8^{\dagger \dagger}$ & $43.2 \pm 6.6$ \\
\hline & Post-exercise & $38.8 \pm 7.4$ & $44.2 \pm 7.3$ & $36.2 \pm 7.7$ & $43 \pm 6.6$ \\
\hline \multirow{2}{*}{ SOD (U/g Hb) } & Rest & $1099.1 \pm 84.7^{* *}$ & $1398.8 \pm 122.6$ & $1029.1 \pm 81.3^{\dagger \dagger}$ & $1209.6 \pm 115.9 * *$ \\
\hline & Post-exercise & $1243.7 \pm 84.4^{\alpha \alpha * *}$ & $1547.4 \pm 112^{\alpha \alpha}$ & $1080.2 \pm 46^{\dagger i}$ & $1484.6 \pm 140.2^{\alpha \alpha}$ \\
\hline \multirow{2}{*}{ GPX (U/g Hb) } & Rest & $69.4 \pm 11.6^{* *}$ & $78.6 \pm 8.6$ & $63 \pm 17.2^{\dagger i}$ & $73.4 \pm 9.3$ \\
\hline & Post-exercise & $79.2 \pm 15.1^{\alpha \alpha *}$ & $91.4 \pm 4.2^{\alpha \alpha}$ & $71.1 \pm 17.2^{\dagger \dagger}$ & $82.9 \pm 12.6^{\alpha \alpha *}$ \\
\hline \multirow{2}{*}{ GR (U/g Hb) } & Rest & $6.5 \pm 1.3^{*}$ & $7.7 \pm 0.8$ & $6.1 \pm 0.6$ & $6.4 \pm 0.8^{*}$ \\
\hline & Post-exercise & $7.5 \pm 1.5 * \alpha$ & $8.9 \pm 1.1^{\alpha \alpha}$ & $6.6 \pm 0.8$ & $6.4 \pm 0.9^{*}$ \\
\hline \multirow{2}{*}{ MDA ( $\mu \mathrm{mol} / \mathrm{l})$} & Rest & $0.53 \pm 0.15 * \dagger$ & $0.33 \pm 0.15$ & $0.59 \pm 0.13^{\dagger \dagger}$ & $0.75 \pm 0.26 * *$ \\
\hline & Post-exercise & $0.62 \pm 0.19 * * \dagger$ & $1.05 \pm 0.18^{\alpha \alpha}$ & $0.79 \pm 0.25^{\alpha \alpha \dagger}$ & $0.98 \pm 0.26^{\alpha \alpha}$ \\
\hline \multicolumn{6}{|c|}{${ }^{*}$ : Significantly different from YA $(p<0.05),{ }^{*} *$ : Significantly different from $Y A(p<0.01)$} \\
\hline \multicolumn{6}{|c|}{ \#: Significantly different from YS ( $p<0.05)$, \#\#: Significantly different from YS $(p<0.01)$} \\
\hline \multicolumn{6}{|c|}{ †: Significantly different from OA ( $p<0.05)$, i†: Significantly different from OA $(p<0.01)$} \\
\hline \multicolumn{6}{|c|}{$\alpha$ : Significantly different from resting condition $(p<0.05),{ }^{\alpha \alpha}$ : Significantly different from resting condition $(p<0.01)$} \\
\hline
\end{tabular}

Regarding peroxidation level, our results showed a higher resting MDA level in OA $(p<0.01)$ and lower level in YA $(p<0.05)$ as compared to the two sedentary groups.

\section{Effects of acute exercise}

Following the incremental exercise test, and for all participants, exercise has no effect on non-enzymatic antioxidant levels (ascorbic acid and $\alpha$-Tocopherol). For the OS group, no significant changes in antioxidant enzyme activities were observed. A significant increase in SOD and GPX activities was observed in YS, YA and OA during the post-exercise period $(p<0.01)$. However, GR activity increased only in YS and YA ( $p<0.01$ and $p<0.05$, respectively). In addition, GPX and GR activities were higher in YA during the recovery period $(p<0.05)$.

Following the incremental exercise test, and except for the YS group, an increase in MDA level was observed in YA $(+236 \%)$, OS $(+33 \%)$ and $O A(+30 \%)(p<0.01)$. Compared to the two sedentary groups, YA and OA have a higher post-exercise MDA level $(p<0.01$ and $p<0.05$, respectively).

\section{Discussion}

To the best of the authors' knowledge, this is the first study to explore the influence of aging and physical fitness levels on both an- 
tioxidants and lipid peroxidation level. The present study demonstrates that among healthy older adults, increased physical fitness could restore the aging-associated decrease in antioxidant defenses. Also, although regular physical activity improves antioxidant levels in both age groups, OA subjects have lower antioxidant defense levels than YA participants, which could indicate that the beneficial effects of regular physical activity could be impaired by the aging process.

Our study has demonstrated that the $\alpha$-Tocopherol level at rest was higher in active groups (YA and OA) as compared to sedentary groups. There are two possibilities to explain this finding. Firstly, this higher level of $\alpha$-Tocopherol observed in YA could be related to their higher vitamin E intake as noted in > Table 2. In fact, several studies have demonstrated a close connection between plasmatic $\alpha$-Tocopherol level and dietary vitamin E intake [15, 23]. Secondly, regular physical activity may increase the $\alpha$-Tocopherol level in YA and OA. However, up to now, the mechanism explaining how physical activity regulates $\alpha$-Tocopherol expression is unknown. One possible explanation is that active subjects could maintain a higher level of $\alpha$-Tocopherol in order to counteract the lipid peroxidation induced by the free radical increase during physical activity sessions.

The present study results also showed that aging could modulate antioxidant enzyme activities according to the physical fitness level of the subjects. In fact, as reported in > Table 3, aging does not affect resting antioxidant activities in sedentary groups (YS and OS). However, in physically active groups, YA presented significant higher antioxidant activities (SOD and GR). In addition, except in OS, we showed an increase in antioxidant activity during the postexercise period. However, lipid peroxidation levels increased in all groups at post-exercise. These data report three major findings.

First, the lack of changes in enzymatic activities (SOD, GPX and $G R$ ) in OS during the recovery period suggests that aging could impair antioxidant enzyme efficiency to scavenge ROS produced during exercise. An alteration of antioxidant gene transcription factors (NF-kB and AP-1) [27] and/or a decrease in antioxidant enzyme protein content with aging [33] could explain these results.

Second, similar levels of antioxidant enzymes between YS and OA may indicate that regular physical activity could restore agerelated impairment in antioxidant defenses. Safdar et al. [40] investigated MnSOD activity and MnSOD protein content in young sedentary and older active subjects. They did not report a difference in MnSOD activity between the two groups, although the MnSOD protein content was lower in the older active group. These data could indicate that regular physical activity may induce post-translational modification of antioxidant proteins that could maintain better antioxidant enzyme activities in older active subjects.

Third, the comparison of YA to OA revealed that young subjects have higher antioxidant enzyme activities at rest and during the post-exercise period. These data may indicate that regular physical activity effects on antioxidant enzymes could be impaired by the aging process. Two possibilities have to be considered to explain these results: First, aging could induce an alteration of the redox-sensitive transcription factors [27]. In fact, transcription factors like NF-Kb and AP-1 are present in the promoter of the human enzymatic antioxidant gene and therefore play an important role in antioxidant enzyme activity. Likewise, many studies using ani- mal models have reported an alteration in antioxidant gene transcription factors (NF-Kb and AP-1) with aging [5]. Second, aging could induce post-transcriptional modifications that may impair antioxidant enzyme adaptation to regular physical activity. In this context, Lambertucci et al. [26] investigated the mRNA level and enzyme activity of GPX in young trained and old trained rats. The authors reported higher GPX enzyme activity in young trained rats, whereas the mRNA level was higher in the old trained rats. They concluded that the response of antioxidant enzyme activities in older rats exposed to regular physical activity is less pronounced than the increases in their mRNA levels. This process may occur due to a decrease in translational efficiency with aging [19]. Therefore, post-transcriptional inhibition of antioxidant enzymes was possibly induced by aging. According to these results, these modifications with aging could impair antioxidant enzyme responses to regular physical activity.

On the other hand, our results showed that the resting MDA level was higher in OA and lower in YA compared to the two sedentary groups. These results suggest that physical activity effects on lipid peroxidation level are modulated by the aging process. Thus, in young subjects, it seems that regular physical activity reduces ROS production [42] and/or reinforces antioxidant defense [3], which could decrease lipid peroxidation damage [34]. In contrast, our results showed that regular physical activity promotes lipid peroxidation in older adults. One possible explanation could be that ROS produced during physical activity sessions in OA exceeds their antioxidant capacity. This hypothesis can be supported by the fact that aging is associated with a failure of the mitochondrial respiratory chain in older adults promoting a greater release of free radicals [28]. On the other hand, exercise-induced inflammation reactions were previously demonstrated [25] and some reports showed that aged individuals presented a considerable inflammatory status when compared to young adults [45]. Thus, post-exercise inflammatory reactions could also contribute to the elevation of lipid peroxidation levels in older adults [35]. Lastly, aging is characterized by a decrease in repair/turnover of oxidative damaged biomolecules [13,38], which could also enhance lipid peroxidation damage in active older adults.

\section{Methodological Limitations}

To the best of the authors' knowledge, this is the first study to explore the influence of aging and physical fitness levels on both antioxidant and lipid peroxidation level. However, some limitations inherent to the experimental protocol of this study warrant mention. Firstly, we categorized our participants into two groups of physical fitness based on data collected from the physical activity questionnaire. Although the physical activity questionnaire method was used in several previous studies $[6,46]$, the use of an objective measure of daily physical activity, like accelerometry, would have allowed a better idea about the lifetime physical activity of our participants. Secondly, we only measured selected biomarkers of oxidative stress and did not include an exhaustive list of potential markers ( $F_{2}$-isoprostanes, 8-OHdG, etc.). Although the selection of biomarkers recorded in the present study is well able to characterize oxidative stress status, the inclusion of additional biomarkers may have strengthened the conclusions. Thirdly, according to 
age and the physical fitness level of each group, the MAP measured during the exhaustive exercise was different between groups, which can induce a methodological bias. However, the choice of this model of exercise stems from the fact that all participants could attain their $\mathrm{VO}_{2 \max }$, that is to say, their maximal physical capacity. So if the absolute MAP was different, the relative intensity of exercise of each participant was equal considering that each participant attained his or her maximum $\left(\mathrm{VO}_{2 \mathrm{max}}\right)$. In addition, the standardized incremental levels according to age and physical fitness of each subject, as recommended by the literature [9], permitted attaining a similar exercise duration between groups. So exhaustive incremental exercise permits (i) comparing groups of different ages and physical fitness levels at a relatively similar intensity of exercise $\left(\mathrm{VO}_{2 \max }\right)$, and (ii) standardizing exercise duration in spite of different ages and physical fitness levels.

\section{Conclusion}

The data of this study suggest that aging affects antioxidant activity and lipid peroxidation levels in both sedentary and active individuals. Regular physical activity improves antioxidant defenses and lowers lipid peroxidation level in young more than in aged individuals, which indicates that the beneficial effects of regular physical activity could be impaired by the aging process. Additionally, elderly physically active individuals show antioxidant activity and lipid peroxidation levels similar to young sedentary subjects, emphasizing the importance of regular physical activity to decelerate the aging-associated impairment process. From a practical point of view, these results suggest that regular physical activity induces beneficial effects in oxidant/antioxidant balance in young and older subjects. Consequently, physical activity has to be continued to maintain these health benefits as long as possible. Cycling exercise programs are probably an effective way, but the minimum training load needed to simply maintain rather than increase this antioxidant protection remains to be determined in both young and older subjects.

\section{Conflict of Interest}

The authors have no conflict of interest to declare.

\section{References}

[1] ACSM. Guidelines for exercise testing and prescription. Philadelphia: Lippincott Williams \& Wilkins; 2005

[2] Arikawa AY, Thomas W, Gross M, Smith A, Phipps WR, Kurzer MS, Schmitz KH. Aerobic training reduces systemic oxidative stress in young women with elevated levels of F2-isoprostanes. Contemp Clin Trials 2013; 34: 212-217

[3] Azizbeigi K, Azarbayjani MA, Peeri M, Agha-alinejad H, Stannard S. The effect of progressive resistance training on oxidative stress and antioxidant enzyme activity in erythrocytes in untrained men. Int J Sport Nutr Exerc Metab 2013; 23: 230-238
[4] Baecke JA, Burema J, Frijters JE. A short questionnaire for the measurement of habitual physical activity in epidemiological studies. Am J Clin Nutr 1982; 36: 936-942

[5] Bar-Shai M, Carmeli E, Ljubuncic P, Reznick AZ. Exercise and immobilization in aging animals: the involvement of oxidative stress and NF-kappaB activation. Free Radic Biol Med 2008; 15: 202-214

[6] Bori Z, Zhao Z, Koltai E, Fatouros IG, Jamurtas AZ, Douroudos II, Terzis G, Chatzinikolaou A, Sovatzidis A, Draganidis D, Boldogh I, Radak Z. The effects of aging, physical training, and a single bout of exercise on mitochondrial protein expression in human skeletal muscle. Exp Gerontol 2012; 47: 417-424

[7] Bouzid MA, Hammouda O, Matran R, Robin S, Fabre C. Changes in oxidative stress markers and biological markers of muscle injury with aging at rest and in response to an exhaustive exercise. PLoS One 2014; 9: e90420

[8] Bouzid MA, Filaire E, McCall A, Fabre C. Radical oxygen species, exercise and aging: an update. Sports Med 2015; 45: 1245-1261

[9] Sperlich PF, Holmberg HC, Reed JL, Zinner C, Mester J, Sperlich B. Individual versus standardized running protocols in the determination of VO2max. J Sports Sci Med 2015; 14: 386-393

[10] Dill DB, Costill DL. Calculation of percentage changes in volumes of blood, plasma, and red cells in dehydration. J Appl Physiol 1974; 37 : 247-248

[11] Dupin H, Abraham J, Giachetti I. (eds.) Apports nutritionnels conseillés pour la population française. Paris: Tec et Doc; 2001

[12] El Abed K, Rebai H, Bloomer RJ, Trabelsi K, Masmoudi L, Zbidi A, Sahnoun Z, Hakim A, Tabka Z. Antioxidant status and oxidative stress at rest and in response to acute exercise in judokas and sedentary men. J Strength Cond Res 2011; 25: 2400-2409

[13] Friguet B. Oxidized protein degradation and repair in ageing and oxidative stress. FEBS Lett 2006; 580: 2910-2916

[14] Fulle S, Protasi F, Di Tano G, Pietrangelo T, Beltramin A, Boncompagni $\mathrm{S}$, Vecchiet $\mathrm{L}$, Fano $\mathrm{G}$. The contribution of reactive oxygen species to sarcopenia and muscle ageing. Exp Gerontol 2004; 39: 17-24

[15] Gascón-Via P, Garcia-Closas R, Serra-Majem L, Pastor MC, Ribas L, Ramon JM, Mariné-Font A, Salleras L. Determinants of the nutritional status of vitamin $\mathrm{E}$ in a non-smoking Mediterranean population. Analysis of the effect of vitamin $E$ intake, alcohol consumption and body mass index on the serum alpha-tocopherol concentration. Eur J Clin Nutr 1997; 51: 723-728

[16] Gomes EC, Silva AN, de Oliveira MR. Oxidants, antioxidants, and the beneficial roles of exercise-induced production of reactive species. Oxid Med Cell Longev 2012; 756132

[17] Gomez-Cabrera MC, Domenech E, Viña J. Moderate exercise is an antioxidant: upregulation of antioxidant genes by training. Free Radic Biol Med 2008; 44: 126-131

[18] Harriss DJ, Atkinson G. Ethical standards in sports and exercise science research: 2016 update. Int J Sports Med 2015; 36: 1121-1124

[19] Ho YS, Dey MS, Crapo JD. Antioxidant enzyme expression in rat lungs during hyperoxia. Am J Physiol 1996; 270: L810-L818

[20] Holbrook N], Ikeyama S. Age-related decline in cellular response to oxidative stress: links to growth factors signaling pathways with common defects. Biochem Pharmacol 2002; 64: 999-1005

[21] Hollander J, Fiebig R, Gore M, Ookawara T, Ohno H, Ji LL. Superoxide dismutase gene expression is activated by a single bout of exercise in rat skeletal muscle. Pflugers Arch 2001; 442: 426-434

[22] Hyltander A, Bosaeus I, Svedlund J, Liedman B, Hugosson I, Wallengren O, Olsson U, Johnsson E, Kostic S, Henningsson A, Körner U, Lundell L, Lundholm K. Supportive nutrition on recovery of metabolism, nutritional state, health-related quality of life, and exercise capacity after major surgery: A randomized study. Clin Gastroenterol Hepatol 2005; 3: 466-474 
[23] Illison VK, Rondó PH, de Oliveira AM, D'Abronzo FH, Campos KF. The relationship between plasma alpha-tocopherol concentration and vitamin $\mathrm{E}$ intake in patients with type 2 diabetes mellitus. Int J Vitam Nutr Res 2011; 81: 12-20

[24] Jackson M], McArdle A. Age-related changes in skeletal muscle reactive oxygen species generation and adaptive responses to reactive oxygen species. J Physiol 2011; 589 (Pt 9): 2139-2145

[25] Ji LL. Exercise at old age: Does it increase or alleviate oxidative stress? Ann N Y Acad Sci 2001; 928: 236-247

[26] Lambertucci RH, Levada-Pires AC, Rossoni LV, Curi R, Pithon-Curi TC. Effects of aerobic exercise training on antioxidant enzyme activities and mRNA levels in soleus muscle from young and aged rats. Mech Ageing Dev 2007; 128: 267-275

[27] Lavrovsky Y, Chatterjee B, Clark RA, Roy AK. Role of redox-regulated transcription factors in inflammation, aging and age-related diseases. Exp Gerontol 2000; 35: 521-532

[28] Maklashina E, Ackrell BA. Is defective electron transport at the hub of aging? Aging Cell 2004; 3: 21-27

[29] Michailidis Y, Jamurtas AZ, Nikolaidis MG, Fatouros IG, Koutedakis Y, Papassotiriou I, Kouretas D. Sampling time is crucial for measurement of aerobic exercise-induced oxidative stress. Med Sci Sports Exerc 2007; 39: 1107-1113

[30] Miyazaki H, Oh-ishi S, Ookawara T, Kizaki T, Toshinai K, Ha S, Haga S, Ji $\mathrm{LL}$, Ohno $\mathrm{H}$. Strenuous endurance training in humans reduces oxidative stress following exhausting exercise. Eur ] Appl Physiol 2001; 84: 1-6

[31] Muller FL, Lustgarten MS, Jang Y, Richardson A, Van Remmen H. Trends in oxidative aging theories. Free Radic Biol Med 2007; 43: 477-503

[32] Muthusamy VR, Kannan S, Sadhaasivam K, Gounder SS, Davidson C], Boeheme C, Hoidal JR, Wang L, Rajasekaran NS. Acute exercise stress activates Nrf2/ARE signaling and promotes antioxidant mechanisms in the myocardium. Free Radic Biol Med 2012; 52: 366-376

[33] Oh-ishi S, Kizaki T, Nagasawa J, Izawa T, Komabayashi T, Nagata N, Suzuki K, Taniguchi N, Ohno H. Effects of endurance training on superoxide dismutase activity, content and mRNA expression in rat muscle. Clin Exp Pharmacol Physiol 1997; 24: 326-332

[34] Ortenblad N, Madsen K, Djurhuus MS. Antioxidant status and lipid peroxidation after short-term maximal exercise in trained and untrained humans. Am J Physiol 1997; 272: R1258-R1263

[35] Parise G, Phillips SM, Kaczor ]J, Tarnopolsky MA. Antioxidant enzyme activity is up-regulated after unilateral resistance exercise training in older adults. Free Radic Biol Med 2005; 39: 289-295
[36] Powers SK, Nelson WB, Hudson MB. Exercise-induced oxidative stress in humans: cause and consequences. Free Radic Biol Med 2011; 51: 942-950

[37] Radak Z, Chung HY, Koltai E, Taylor AW, Goto S. Exercise, oxidative stress and hormesis. Ageing Res Rev 2008; 7: 34-42

[38] Radak Z, Zhao Z, Goto S, Koltai E. Age-associated neurodegeneration and oxidative damage to lipids, proteins and DNA. Mol Aspects Med 2011; 32: 305-315

[39] Sacheck JM, Milbury PE, Cannon JG, Roubenoff R, Blumberg JB. Effect of vitamin $E$ and eccentric exercise on selected biomarkers of oxidative stress in young and elderly men. Free Radic Biol Med 2003; 34: 1575-1588

[40] Safdar A, Hamadeh M], Kaczor J], Raha S, Debeer J, Tarnopolsky MA. Aberrant mitochondrial homeostasis in the skeletal muscle of sedentary older adults. PLoS One 2010; 5: e1077

[41] Simar D, Malatesta D, Badiou S, Dupuy AM, Caillaud C. Physical activity modulates heat shock protein-72 expression and limits oxidative damage accumulation in a healthy elderly population aged 60-90 years. J Gerontol A Biol Sci Med Sci 2007; 62: 1413-1419

[42] Starnes JW, Barnes BD, Olsen ME. Exercise training decreases rat heart mitochondria free radical generation but does not prevent $\mathrm{Ca} 2+$-induced dysfunction. J Appl Physiol 2007; 102: 1793-1798

[43] Syu GD, Chen HI, Jen C]. Severe exercise and exercise training exert opposite effects on human neutrophil apoptosis via altering the redox status. PLoS One 2011; 6: e24385

[44] Tanaka H, Monahan KD, Seals DR. Age-predicted maximal heart rate revisited. J Am Coll Cardiol 2001; 37: 153-156

[45] Toft AD, Jensen LB, Bruunsgaard H, Ibfelt T, Halkjaer-Kristensen J, Febbraio M, Pedersen BK. Cytokine response to eccentric exercise in young and elderly humans. Am J Physiol 2002; 283: C289-C295

[46] Traustadóttir T, Davies SS, Su Y, Choi L, Brown-Borg HM, Roberts L] 2nd, Harman SM. Oxidative stress in older adults: Effects of physical fitness. Age (Dordr) 2012; 34: 969-982

[47] Viña J, Borras C, Abdelaziz KM, Garcia-Valles R, Gomez-Cabrera MC. The free radical theory of aging revisited: the cell signaling disruption theory of aging. Antioxid Redox Signal 2013; 19: 779-787

[48] Voorrips L, Ravelli A, Dongelmans P, Deurenberg P, Staversen W. A physical activity questionnaire for the elderly. Med Sci Sports Exerc 1991; 23: 974-979

[49] Wei YH, Lee HC. Oxidative stress, mitochondrial DNA mutation, and impairment of antioxidant enzymes in aging. Exp Biol Med (Maywood) 2002; 227: 671-682 